affective words. We used a mixed quasi-experimental design with age ( 2 ; young, old) and posture ( 2 ; upright, stooped) as between-subjects factors and word valence ( 3 ; positive, negative, neutral) as a within-subjects factor. Using a sample of 42 younger and 24 older adults, a repeated measures ANOVA revealed that although the 3 -way interaction was non-significant $(\mathrm{p}>.05)$, the interactions between age and posture $(\mathrm{F}(1,62)=4.162, \mathrm{p}=.046, \mathrm{np} 2=.063)$ as well as age and word valence were significant $(\mathrm{F}(2,124)=6.204, \mathrm{p}=.003$, $\eta \mathrm{p} 2=.091)$. Younger adults displayed a positivity bias in both postural conditions; this effect was absent in both older adult groups. Furthermore, older adults performed worse overall and exhibited a negativity bias in stooped postures. Findings will be discussed based on current theoretical work on age-related positivity and mind-body integration in later life.

\section{INVESTIGATING RELATIONSHIPS BETWEEN HYPERTENSION, SLEEP, AND COGNITIVE RISK IN A RURAL, ETHNICALLY DIVERSE COHORT}

L. Wiese ${ }^{1}$, P. Abdul-Akbar ${ }^{2}$, 1. Florida Atlantic University, 2. College of Science, Florida Atlantic University

Currently, there are over 47 million people worldwide who have been diagnosed with dementia. The risk for dementia is magnified in underserved populations (Mattos, et al, 2017). Recent studies point to the significance of cardiovascular disease and sleep patterns as factors that can increase dementia risk (ADI, 2017). The purpose of this descriptive study was to investigate the relationship between hypertension, sleep deprivation, and risk of cognitive impairment in a rural, underserved, and ethnically and racially diverse population. The sample $(\mathrm{n}=51)$ was drawn from three subsidized independent older adult living facilities that are home to 218 residents in a rural Florida area known as the Glades. This sample is largely comprised of immigrants and retired farmworkers, of which $88 \%$ are African American or Hispanic, and $11 \%$ are Afro-Caribbean. The data collection consisted of (1) the Sociodemographic Survey, (2) a Likert-type scale regarding sleep patterns, (3) a questionnaire addressing family and personal history of cardiovascular disease and (4) blood pressure measurements. Findings included that the majority of participants were not at risk for hypertension (73\%) or cognitive impairment $(76 \%)$, but those with hypertension risk correlated significantly $(\mathrm{p}=.01)$ with cognitive impairment risk $(\mathrm{r}=.396)$. Contrary to recent findings suggesting that the quality of sleep may increase risk for hypertension and dementia, sleep quality in this small cohort did not significantly influence either blood pressure or cognition. These findings suggest that examining potential cultural influences on sleep and hypertension as risk factors for dementia is needed.

\section{LONGITUDINAL RELATIONSHIP BETWEEN COGNITIVE COSTS AND NEED FOR COGNITION}

A. Lothary, C. Growney, T. Hess, North Carolina State University

Selective Engagement Theory (SET; Hess, 2014) proposes increases in the costs associated with engaging in cognitive activities as we age. These costs have been studied as effort expended and measured through cardiovascular responsivity. The effort required to maintain a certain level of performance may be higher for some than others, and is thought to influence intrinsic motivation to engage in cognitively demanding activities. We sought to explore the longitudinal associations between individual differences in engagement costs and changes in need for cognition (NFC; Cacioppo et al., 1982), which assesses the motivation to engage in effortful cognitive activities. As part of a longitudinal study, 140 older adults (aged 64-81 at time 1) completed a challenging cognitive task while their systolic blood pressure was recorded continuously. NFC was also assessed at that time and then again 2 years later (time 2). We hypothesized that costs at time 1 , represented by the linear function of systolic blood pressure responses over levels of task demands, would be predictive of NFC at time 2 . We found that when controlling for NFC and health at time 1, costs significantly predicted NFC at time $2(\mathrm{p}<.05)$. Specifically, those who experienced higher costs associated with cognitive engagement exhibited reductions in levels of NFC over time, in line with SET. These results suggest the increased costs of engaging in cognitively demanding activities associated with aging may negatively affect desire to engage in such activities, with a potentially detrimental impact on cognitive health.

\section{LOSS OF ACTIVITY AND SOCIAL SUPPORT MEDIATES EFFECT OF COGNITIVE IMPAIRMENT ON WELL-BEING IN THE VERY OLD}

\section{R. Kaspar, University of Cologne, Germany}

Reduced levels of subjective well-being (SWB) are consistently reported in persons with cognitive impairment. Little is known about consequences in everyday life of affected individuals that drive this association, particularly in the general population of the very old. Problems with orientaton have been found to restrict the use of out-of-home space in early dementia and mild cognitive impairment (MCI). Similarly, patterns of social support are transformed by the own current vulnerability and the prospect of increasing support needs. Hence, we expect some of the effect of cognitive impairment on SWB to be mediated by a loss of opportunity for positive experience such as out-of-home activity and support of relevant others. Subjects are part of a representative sample of the very old $(80+)$ from the NRW80+ study and show age-adequate cognitive functioning $(n=981)$, MCI $(n=230)$ or early Alzheimer's disease $(n=139)$ according to DemTect screening. SWB is measured by positive affect, valuation of life, depressive symptoms and life satisfaction. Social support is measured by the frequency of received and provided financial, instrumental and emotional support. Participation in 13 leisure activities is aggregated as an indicator of out-ofhome activity. Estimated prevalence of MCI and beginning $\mathrm{AD}$ in the studied population is $16.0 \%$ and $8.7 \%$, respectively. Impaired cognitive function is found to impact on SWB primarily via losses in out-of-home activity and support provided for significant others (total indirect effect: .158**;, direct effect: $.099 * * *$ ). Results call for a stronger emphasis on socio-emotional consequences of cognitive decline to understand well-being in the very old.

\section{NEUROPSYCHIATRIC SYMPTOMS AS A DISTINGUISHING FACTOR BETWEEN MEMORY DIAGNOSES}

H. Rouse ${ }^{1}$, J. Schinka ${ }^{2}$, B. Small ${ }^{3}$, 1. University of South Florida, 2. School of Aging Studies, University of South 
Florida, 3. School of Aging Studies, University of South

Florida, Tampa, FL, USA

With an aging population, identifying factors that allow persons with cognitive disorders to be differentiated from those who are cognitively health is imperative. Studies have identified that neuropsychiatric symptoms (NPS) may enhance our ability to differentiate memory diagnoses in older adults. Several hypotheses have predicted that presence of NPS in a cognitively healthy population may result in progression to a memory diagnosis, and that presence of NPS within those with a memory diagnosis may predict faster progression into dementia. The sample was recruited from the Florida Alzheimer's Disease Center, of whom 317 were cognitively healthy, 247 were classified with mild cognitive impairment amnestic (MCI-A) or non-amnestic (MCI-NA), and 127 were diagnosed with dementia. The Neuropsychiatric Inventory assessed the presence of 12 NPS. The results indicated that persons with MCI-A, MCI-NA, and dementia were significantly more likely to have NPS (all p's<.05) compared to those with no memory diagnosis $(\mathrm{p}<.05)$. For individuals with MCI-A, there were significant differences in endorsement of delusions, agitation, depression, anxiety, apathy, disinhibition, irritability, and appetite changes (all p's<.05). For individuals with MCI-NA, there were significant differences in those who endorsed delusions, hallucinations, agitation, depression, anxiety, apathy, motor disturbances, nighttime disturbances, and appetite changes (all p's $<.05$ ). Finally those with dementia, had significant differences in endorsement of delusions, hallucinations, agitation, apathy, disinhibition, motor disturbances, and appetite changes (all p's<.05). The current paper demonstrates that endorsement of specific NPS can help us in identifying specific memory diagnoses. Future research should explore predictive value of NPS scores for memory diagnoses.

\section{OLDER ADULTS' EVERYDAY MEMORY BELIEFS AND ATTRIBUTIONS: A QUALITATIVE INTERVIEW ANALYSIS}

\section{E. Lustig, C. Hertzog, A. Pearman, A. Waris, Georgia} Institute of Technology

How older adults describe the causal factors involved in memory successes and failures interplays with their beliefs about their own memory abilities and the behaviors they engage in to support their everyday remembering. Questions remain about how their beliefs and attributions about memory promote or deter the use of everyday memory strategies. This study evaluated these issues through qualitative coding of 26 individually tailored, semi-structured interviews with older adults ages $62-83(\mathrm{M}=69.5$, $\mathrm{SD}=5.72$ ) concerning their everyday remembering. The interviews were focused on how older adults implemented memory strategies, how efficacious these procedures were in helping them achieve their goals, and how they conceptualized their behaviors. The interviews provided a greater understanding of the context and depth in which memory failures occurred. We focus on one of the major emergent themes, beliefs and attributions about memory. Habits and partially-structured routines were not typically directly attributed as the causes of successful daily remembering. However, these behaviors were mentioned when people were asked about how they accomplished tasks and goals.
Participants also reported that important things would be remembered, regardless of strategy use or memory-supportive behaviors, and stated that forgetting was usually about inconsequential things. This "importance" belief was observed in tandem with reports of forgetting significant events (e.g. a spouse's birthday). Finally, when everyday memory issues were acknowledged, they were often attributed to age-related declines in memory and viewed as being inherently uncontrollable.

\section{PHOTOJOURNALISM REDUCES CAREGIVER BURDEN AND DEPRESSION IN DEMENTIA CAREGIVERS}

M. Kovaleva ${ }^{1}$, W. Wharton ${ }^{1}$, F. Epps ${ }^{2}$, L. Bridwell ${ }^{3}$, R. Tate ${ }^{4}$ C. Dorbin ${ }^{1}$, K. Hepburn ${ }^{1}$, 1. Emory University,

2. Georgia State University, 3. Emory University Rollins

School of Public Health, 4. Emory College Center for

Creativity \& Arts

Most persons living with dementia rely on informal caregivers. This study aimed to describe a pilot photojournalism program for 10 caregivers of persons living with dementia with respect to caregivers' mood, depressive symptoms, and experience. A professional photographer taught photography in a four-session workshop. Caregivers photographed what they saw as representative of their experience and discussed the photographs in class. Quantitative exploration consisted of pre- and post-program comparison in caregiver burden (Zarit Burden Interview) and depression (Patient-Reported Outcomes Measurement Information System - Short Form Depression). Qualitative exploration included investigators' observations, viewing photographs, and recording caregivers' comments on their photographs. For participants with preand post-program data $(\mathrm{N}=5)$, caregiver burden decreased significantly $(\mathrm{p}=0.037)$ and depression decreased non-significantly $(p=0.066)$. Statistically non-significant but clinically meaningful reductions in caregiver burden and depression were attained. Analysis of photographs and caregivers' accompanying commentaries yielded three themes: caregivers' emotional well-being, shared experiences, and classroom engagement. Qualitative data demonstrated caregivers' satisfaction with the program and their ability to express their experiences in a new creative format and discuss their challenges, using photographs as daily life examples. These results demonstrated efficacy of this photojournalism intervention in the reduction of caregiver burden and depression. Caregivers could reflect upon their work with other participants and get their feedback. Future studies should employ a larger sample size and test more patient- and caregivercentered variables. Provided their positive effect on dyads, these programs may be more widely adopted by healthcare, educational, and community agencies, such as museums.

\section{PRACTICE EFFECTS ON LIST LEARNING ARE ASSOCIATED WITH LATER SCORES ON GLOBAL COGNITION}

T. Atkinson ${ }^{1}$, R. Andel ${ }^{2}, 1$. University of South Florida, 2.

University of South Florida, School of Aging Studies

While often considered a confound in longitudinal studies, practice effects (PEs) can be useful in predicting change in performance. We assessed whether PEs would predict global cognitive scores across three annual (9-15 months) 